\title{
Puzzle-solving in Psychology: the Neo-Galtonian vs. Nomothetic Research Focuses
}

\author{
Stéphane Vautier ${ }^{\mathrm{a}}$, Émilie Lacot ${ }^{\mathrm{a}}$, Michiel Veldhuis ${ }^{\mathrm{b}}$ \\ ${ }^{a}$ University of Toulouse \\ ${ }^{b}$ Utrecht University
}

\begin{abstract}
We compare the neo-Galtonian and nomothetic approaches of psychological research. While the former focuses on summarized statistics that depict average subjects, the latter focuses on general facts of form 'if conditions then restricted outcomes'. The nomothetic approach does not require quantification as a convenient way of statistical modeling. The nice feature of a general fact is its falsifiability by the observation of a single case. Hence, as a clear sense of scientific error is re-introduced in the research paradigm, we detail two kinds of puzzle-solving: repairing general facts by contraction or by expansion of the initial conditions. This style of research does not require that researchers depend on highly skilled engineers in data analysis, as the very structure of a general fact can be established by scrutinizing a contingency table.
\end{abstract}

Keywords: methodology, measurement error, general fact, falsifiability

The present article contrasts two research focuses in Psychology. As these research focuses define two classes of goals, we will call them paradigms. The first one has been called neo-Galtonian by Danziger $(1987,1990)$ and is most generally used in mainstream methodology in psychology, while the second one can be properly called nomothetic, as argued by Lamiell (1998) and Vautier (2011, 2013), and constitutes a blind spot of psychological research. Let $P_{1}$ and $P_{2}$ denote neo-Galtonian and nomothetic research, respectively. To describe concisely these two paradigms, the mathematical concept of a relation will be useful. ${ }^{1} \mathrm{~A}$ (binary) relation from a set $A$ on a set $B$ is defined

\footnotetext{
${ }^{1}$ According to French philosopher of science Gilles-Gaston Granger (1995), "Scientific
} 


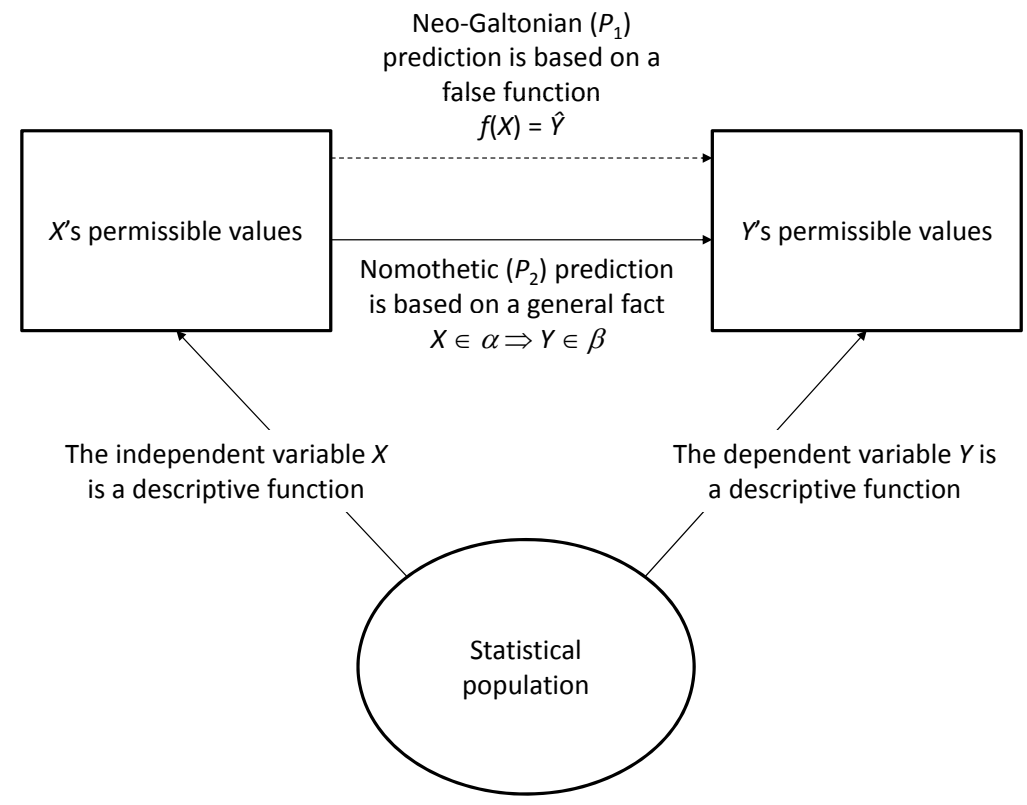

Figure 1: The common statistical structure of neo-Galtonian and nomothetic forms of prediction, and their specific features.

by a set of ordered pairs $(a, b)$ such that $a$ is in $A(a \in A)$ and $b$ is in $B$. (This set is called the graph of the relation.)

Whereas the $P_{1}$ 's researcher is used to being satisfied with relations that emerge from aggregates of persons but are logically irrelevant to depict phenomena at the scale of single persons (see, e.g., Danziger, 1987; Krause, 2011; Lamiell, 2003, 2013; Molenaar, 2004), the $P_{2}$ 's researcher inspects the same data to discover a special case of relations, what Vautier (2013) calls general facts, which are, by definition, true for any person. As depicted in Figure 1 , an easy to spot difference would be that, whereas $P_{1}$ focuses on expected point-values, $P_{2}$ focuses on a necessary set of values. $P_{1}$ 's slogan may be expressed as follows:

$$
Y=f(X)+E
$$

knowledge based on experience always consists of constructing schemas or abstract models of this same experience and uses the relations between the abstract elements of these models to deduce-through logic and mathematics-properties that correspond with sufficient precision to directly observable empirical properties." (p. 95, authors' translation) 
where $Y$ is the dependent variable, $X$ is the independent variable and describes initial conditions, $f$ is a function of these conditions, and $E$ denotes a random component that obeys a convenient probability law. The crucial concept here is that of a function, that is, the special case of a relation such that $f(X)$ is a single value ${ }^{2}$.

Contrastingly, $P_{2}$ 's slogan can be formulated as follows:

$$
X \in \alpha \Rightarrow Y \in \beta,
$$

where $\alpha$ and $\beta$ are non-empty strict subsets of $X$ and $Y$ 's sets of permissible values (the respective codomains of $X$ and $Y$ ). Thus, although $P_{2}$ does not preclude a functional relationship, it does not expect it. What is especially expected is that the descriptive device provided by $X$ and $Y$ 's codomains will suggest that there is a least one subset of initial conditions which works as a sufficient condition for a strict subset of $Y$ 's codomain.

What is at stake is to move freely from one research paradigm to another, instead of defining opposite and mutually ignoring camps. Within $P_{1}$, psychologists are used to manipulate propositions pertaining to 'constructs' (Cronbach \& Meehl, 1955); for critical views, see Maraun (1998) and Michell (2013). As a corollary, $P_{1}$-psychologists are trained to accept the institutional division of labour between the psychologist, whose expertise pertains to substantive theory, that is, the realm of constructs or the "nomological network', and the statistician (or psychometrician), whose expertise deals with data analysis. A catastrophic consequence of this division of labor is that the sense of scientific error within the discipline becomes more and more esoteric or even socially irrelevant (see, e.g., Borsboom, 2006). This is why, if psychologists are motivated to make a science of their discipline (see, e.g., Borsboom et al., 2009; Lilienfeld, 2010; Vautier, 2011), they have to take intellectual responsibility for its epistemology and methodology. Within $P_{2}$, division of labor is superfluous. However, the required intellectual style obeys Monsieur Teste's injunction: "Always demand proof, proof is the elementary courtesy that is anyone's due" (Valéry, 1973, p. 65).

In the first section of the present article, it is argued that $P_{1}$ 's slogan mimics functional prediction in the natural sciences, but the price to be paid

\footnotetext{
${ }^{2} \mathrm{~A}$ (statistical) variable is also a relation, which is defined from the set of a populationcalled its domain-on the set of its admissible values-its codomain. The population is the domain of $X$ and $Y$. The function $f$ is a relation from the codomain of the independent variable $X$ on the codomain of the dependent variable $Y$.
} 
is that the sense of approximation that characterizes the natural sciences is lost. $P_{2}$ 's slogan rejects the functional imperative by acknowledging irreducible indetermination of prediction and instead hypothesizes restricted approximation. The second section opposes the infalsifiability of predictive statements in $P_{1}$ to the falsifiability of predictive statements in $P_{2}$. The third section exposes two kinds of puzzle-solving $P_{2}$-researchers have to deal with.

\section{Prediction: Restricted vs. Unrestricted Range of Approxima- tion}

Let us take Kuhn's (1996) words to get a sense of what empirical approximation means in this context:

Perhaps the most striking feature of the normal research problems we have just encountered [in the physical sciences] is how little they aim to produce major novelties, conceptual or phenomenal. Sometimes, as in a wave-length measurement, everything but the most esoteric detail of the result is known in advance, and the typical latitude of expectation is only somewhat wider. Coulomb's measurements need not, perhaps, have fitted an inverse square law; the men who worked on heating by compression were often prepared for any one of several results. Yet even in cases like these the range of anticipated, and thus of assimilable, results is always small compared with the range the imagination can conceive. And the project whose outcome does not fall in that narrower range is usually just a research failure, one which reflects not on nature but on the scientist. (p. 35)

In other terms, the paradigmatic slogan of normal quantitative science expresses as

$$
y=f(x)+\epsilon,
$$

where $y$ and $x$ are quantitative variables, $f$ is a numerical function, and $\epsilon$ denotes an approximation component within a restricted $100 \%$ confidence interval. By 'restricted', we mean that the range of the expected outcomes enables the researcher to eliminate a wide subset of logically possible outcomes that the measurement procedure allows.

For example, we can determinate that Paul's height today lies between 1.74 and $1.76 \mathrm{~cm}$, and this range of approximation is compatible with the 
claim that the probability to find that, in suitable measurement conditions, Paul's height today is not in this interval is null. If it was the case, such a finding would merely reflect a measurement aberration-not a measurement error. In $P_{1}$-psychology, such a finding would be qualified as measurement error. To make the point clear, it suffices to remark that the random component in Equation (1) takes its values in the set of the possible values of the dependent variable. Usually, this variable is given a Gaussian probability density function, which is valued in $]-\infty,+\infty[$. Hence, this approach to prediction precludes aberrant outcomes: any outcome is possible.

It is a striking paradox that quantitative psychology, while having adapted its paradigmatic slogan from Equation (3) to Equation (1), has conceded the epistemological principle of unpredictability of its dependent variables. Consequently, the concept of (restricted) approximation used in the natural sciences has been lost. The prediction $\hat{Y}=f(X)$ becomes a better expectation, yet preserving practical utility for decision making in mass settings. Imitation of quantitative laws saves the form of approximative prediction in Equation (3), as $\hat{Y}$ is a real value, but ratifies total ignorance at the scale of the observation unit since the approximation of prediction is now unrestricted.

The paradigm $P_{2}$ goes one step further, as its typical goal consists in re-introducing the sense of restricted approximation in psychological prediction. There is a double issue at stake. First, if it is acknowledged that $P_{1^{-}}$ psychology is valid only for mass psychology, there is a need for a genuinely general psychological science, i.e., a science valid (and hopefully, sound) for any psychological system. In clinical psychology, in cognitive psychology, in developmental psychology, psychological systems correspond to single persons and not to collectives of persons. Even in social psychology, the unit of analysis is a person whose behavior is analyzed with respect to a group of persons. However, $P_{1}$-psychology admits that the single person is unpredictable. For example, according to Rasch (1960),

Where it is a question of human beings and their actions, it appears quite hopeless to construct models which can be useful for purposes of prediction in separate cases. On the contrary, what a human being actually does seems quite haphazard, none less than radioactive emission. (p. 11)

In the perspective of $P_{2}$-psychology, such a claim deserves verification. Thus, the target of $P_{2}$-psychology consists in predictions with restricted approx- 
imation. Importantly, usefulness of $P_{2}$ 's program for social engineering in mass settings should not be mandatory, as restricted approximation means exactly that what is to be predicted is a strict subset of values (which does not entail that its cardinality is one-in which case the prediction would be a point value). Thus, a priori, psychological phenomena that can be described by psychologists do not warrant the degree to which approximation can be reduced and the fact that approximation is restricted is a starting hypothesis.

Coming back to Equation (1), the aim is to discover initial conditions (pertaining to $X$ ) that warrant bounded residual variability, in such a way that knowing the initial conditions, it would be possible to exclude at least one admissible value of the dependent variable $(Y)$. Such aim involves a reversal of perspective: whereas Equation (1) focuses on the positive, punctual, necessary, and in fact false value $\hat{Y}$ given $X$, Equation (2) focuses on a strict necessary and hopefully true subset $\beta$ given $X . P_{2}$ 's perspective reconciles psychological research and what constitues for us the very wisdom of scientific knowledge, which consists in admitting and recognizing various forms of objective impossibility. Indeed, Equation (2) focuses equivalently on the complement set $\bar{\beta}$ of empirical impossible values given $X$. Let $M(Y)$ denote $Y$ 's codomain (set of admissible values). The existence of $\beta$ implies that of its non-empty complement $\bar{\beta}$ :

$$
M(Y) \backslash \beta=\bar{\beta} .
$$

Thus, $P_{2}$ focuses on a set $\bar{\beta}$ of empirically impossible, although logically possible, events conditionally to given initial conditions identified as $\alpha$, a strict subset of $X$ 's codomain. The reversal of focus from necessity to impossibility echoes Popper's (1959) profound remark: "[natural laws] do not assert that something exists or is the case; they deny it" (p. 48).

In addition, there is no need to postulate that natural laws are quantitative, and the dependent variable $Y$ may be qualitative in "nature"-although the definition of the codomain of any function $Y$ is not natural but cultural (Danziger, 1990). ${ }^{3}$ This is the second issue at stake with adopting $P_{2}$ 's research focus. To sum up, the shift from $P_{1}$ to $P_{2}$ is double: quantitative

\footnotetext{
3 "But in truth scientific psychology does not deal in natural objects. It deals in test scores, rating scales, response distributions, serial lists, and innumerable other items that the investigator does not find but constructs with great care. Whatever guesses are made about the natural world are totally constrained by this world of artifact" (p. 2).
} 
dependent variables are replaced by qualitative ones, and the expected prediction, a point-value, is replaced by a necessary strict subset of values.

\section{Testability of Predictions}

As far as Equation (1) comprises the random component $E$, and given that E's distribution is usually thought of as Gaussian, it is clear that a statement of form (1) denies nothing: given any image of $X$ (or any observed value on $X), Y$ may take any value in its codomain $M(Y)$. Actually, the normal distribution of $E$ is a crude and logically unsuitable approximation because $M(Y)$ is bounded and discrete (e.g., a test scale is a finite numerical series, see Vautier et al., 2011, 2012). The crucial point is that the random variable is defined in such a way that its range of possible values is unrestricted, that is, it implies no impossible values in $M(Y)$. As "it is a fallacy to infer an individual propensity from a statistical analysis of the distribution of a property in a population" (Harré, 2004, p. 7), the random variable $E$ plays the role of an interpretative concept, by contrast to a falsifiable probabilistic hypothesis about residual variability associated with the observable response of a class of observation units from the population. Thus, $P_{1}$ focuses on the appearance of punctual predictability (for the sake of social utility) and loses falsifiability at the level of a class of observation units induced by a given value on $X$.

The paradigm $P_{2}$ focuses on falsifiability, even if it means that punctual predictability may be lost as the primary motivation of the research projectwhich does not entail that punctual estimation is lost if this kind of goal is to be pursued for other non-scientific reasons. The logical form of Equation (2) implies that if it is true, the following statement is true as well:

$$
Y \in \bar{\beta} \Rightarrow X \in \bar{\alpha} \text {. }
$$

As $X$ and $Y$ 's values are observables, Equation (2) is falsifiable: it is logically possible to observe

$$
Y \in \bar{\beta} \& X \in \alpha,
$$

in which case Equation (5) is false and hence Equation (2) is also false.

\section{Puzzle-solving}

Kuhn (1996) describes normal research as puzzle-solving. We have difficulties to figure out how normal research in $P_{1}$ could be described as puzzlesolving at the scale of classes of single cases defined by their common value 
on $X$, as unrestricted approximation is normal. Instead, we understand normal research in $P_{1}$ as a kind of fuzzy process of "translation" of nonempirical ideas-that hardly match the concept of falsifiable theory-into statistical statements that depict the average subject. A characteristic feature of $P_{1}$ is that (substantive) theory is intrinsically disconnected with description (and hence genuine measurement) of real persons. Theoretical explanation consists mainly in elaboration of abstract representations about inobservables and demonstration of confirmatory operationalized evidence at the level of aggregates (Danziger, 1987; Vautier, 2011). In $P_{2}$, theoretical explanation (or invention, Hempel, 1966) has no object until one empirical law has been formulated. We do not know of a single report in psychological literature harboring an empirical statement of form (2). Actually, nomological networks of psychological constructs advocated by Cronbach \& Meehl (1955) work as pre-theoretical or pre-scientific concepts. This is because scientific concepts require empirical regularities to have an empirical meaning. The task of nomothetic $\left(P_{2^{-}}\right)$psychology consists of discovering descriptive approaches able to reveal lawful-and non trivial-phenomena. In the meantime, normal science in nomothetic psychology consists of indexing candidates for lawful statements, and then in indexing and elucidating their associated counterexamples.

To analyse the puzzle-solving's structure in $P_{2}$, it will be useful to detail how the variables $X$ and $Y$ are functions (cf. Figure 1). Their common domain is a discrete set $\Omega=\left\{\omega_{i}, i=1, \ldots\right\}$, and their codomains are $M(X)$ and $M(Y)$, respectively:

$$
\begin{aligned}
& X: \Omega \longrightarrow M(X) \\
& Y: \Omega \longrightarrow M(Y)
\end{aligned}
$$

The important concept is that of the empirical support $\Omega$. The population is a set of observation units of form $\omega=(u, t)$, which denote a person $u$ located in time $t$. There are two possible starting points. The first one is a general fact (Vautier, 2011, 2013), that is, the triplet $\left(\Omega_{n}, \alpha, \beta\right)$ such that

$$
\begin{aligned}
& \exists \alpha \subset M(X), \beta \subset M(Y), \\
& \forall \omega \in \Omega_{n} \subset \Omega, X(\omega) \in \alpha \Rightarrow Y(\omega) \in \beta .
\end{aligned}
$$

In other terms, the triplet $\left(\Omega_{n}, \alpha, \beta\right)$ represents a non tautological fact, of which the generality is bounded to the $n$ objects (or observation units) of the world $\Omega_{n}$. The general fact stipulates sufficient conditions, namely having the property $\alpha$, for having the property $\beta$. 
Table 1: Conjoint distribution of test scores and illness state.

\begin{tabular}{lcc}
\hline Test scores & $i$ & $\neg i$ \\
\hline$[40,71]$ & 26 & 2 \\
{$[72,87]$} & 35 & 51 \\
{$[88,94]$} & 0 & 40 \\
\hline
\end{tabular}

Note. $i=$ illness, $\neg i=$ non illness.

Let us take the example of a memory test the total score from which is used to predict the presence of illness $i$ (Lacot et al., 2011). Thus, $M(X)$ is a univariate set of test scores; specifically, $M(X)=\{40,41, \ldots, 94\}$. And $M(Y)=\{i, \neg i\}$. The data here update Lacot et al.'s data with a sample of 154 participants. Table 1 exhibits the general fact

$$
\forall \omega \in \Omega_{154}, X(\omega) \in[88,94] \Rightarrow Y(\omega)=\neg i,
$$

which suggests a law on the parent population $\Omega$.

Now, a counter-example is the new observation unit $\omega_{155} \in \Omega \backslash \Omega_{154}$ such that

$$
X\left(\omega_{155}\right) \in \alpha \& Y\left(\omega_{155}\right) \in \bar{\beta},
$$

where $\alpha=[88,94]$ and $\bar{\beta}=\{i\}$. Thus, the counter-example $\omega_{155}$ does not falsify the general fact $\left(\Omega_{154}, \alpha, \beta\right)$ but the law $(\Omega, \alpha, \beta)$.

Repairing the law is the $P_{2}$-puzzle. Such problem has the same structure as that of repairing what we call a pseudo-general fact; which constitutes the second possible starting point. In research practice, the latter may be more relevant than the former. A pseudo-general fact is a general fact suffering few counter-examples. Table 1 exhibits the following pseudo-general fact:

$$
\forall \omega \in \Omega_{154}, X(\omega) \in[40,71] \Rightarrow Y(\omega)=i,
$$

which is false because of two counter-examples. Let us note them $\omega_{153}$ and $\omega_{154}$.

Thus, the puzzle is to repair the general fact (11). Levi (1980) suggests two repairing approaches. The first one is contraction of $M(X)$ (see Figure 2, upper panel), while the second one is its expansion (see Figure 2, lower panel). 


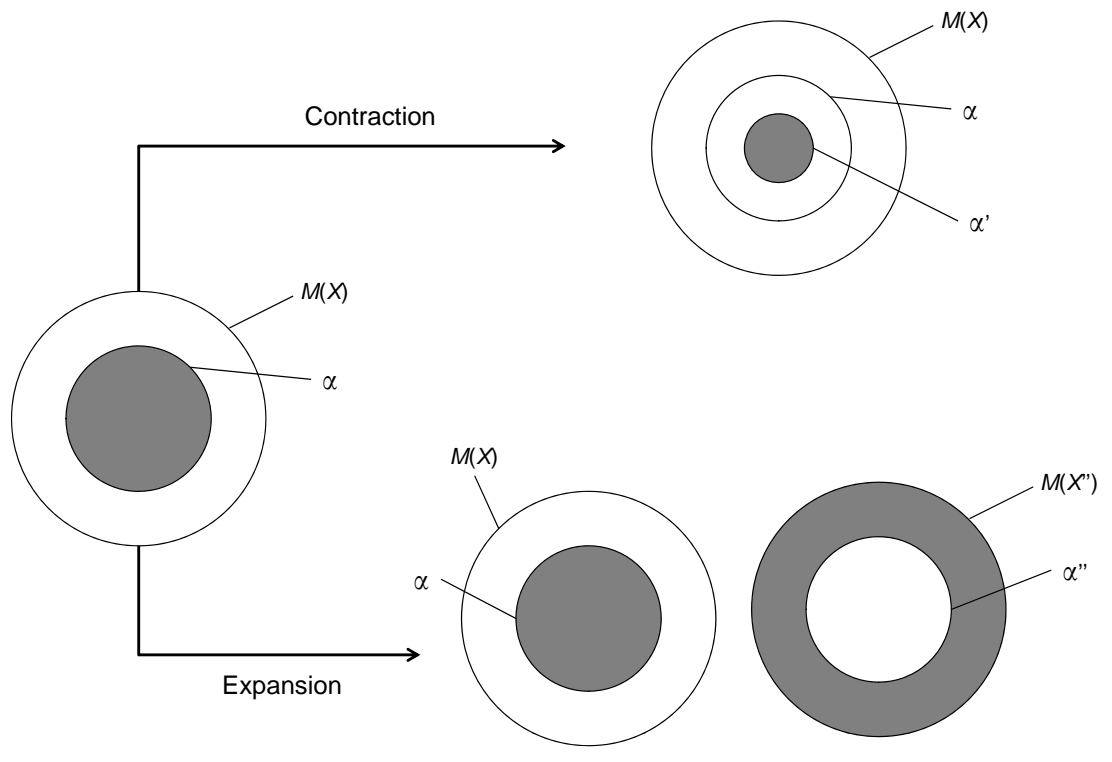

Figure 2: Two strategies for law repairing: Contraction and expansion of the validity domain of a law (in gray, specification of the initial conditions). $\alpha=$ the initial conditions for a law. $\alpha^{\prime}=$ the contracted conditions (where the initial conditions have been adaptedcontracted-to exclude falsifying cases). $\alpha^{\prime \prime}=$ the expanded conditions (where the initial conditions have been expanded, with relevant descriptive variables, to exclude falsifying cases). $M(X)=$ the codomain of $X$ (with the initial conditions). $M^{\prime \prime}(X)=$ the codomain of $X^{\prime \prime}$ (with the expanded conditions). 


\subsection{Contraction}

Contraction aims at restricting the initial conditions to save their sufficient character. Let $\Omega_{n^{\prime}}$ be the set of counter-examples, and $M^{\prime}(X)$ the set of their images by $X$. Coming back to our example, we have to consider that $\Omega_{n^{\prime}}$ is comprised of the elements $\omega_{153}$ and $\omega_{154}$. The data reveal that these two exceptions had test scores of 51 and $67\left(M^{\prime}(X)=\{51,67\}\right)$. The idea consists of removing these specific initial conditions from the statement (11). Let $\alpha^{\prime}=\alpha \backslash M^{\prime}(X)$. Thus,

$$
\forall \omega \in \Omega_{154}, X(\omega) \in \alpha^{\prime} \Rightarrow Y(\omega) \in \beta .
$$

Repairing $\left(\Omega_{154}, \alpha, \beta\right)$ yields $\left(\Omega_{154}, \alpha^{\prime}, \beta\right)$, where $\alpha^{\prime} \subset \alpha$ (and, trivially, $\left.\alpha^{\prime} \neq \emptyset\right)$.

Coming back to the example, we get $\alpha^{\prime}=[40,50] \cup[52,66] \cup[68,71]$. However, the substantive meaning of such repairing has to be assessed. We get no insight in removing the specific values 51 and 67 from the initial conditions because we are not ready here to question the assumption that the test scores measure an ordinal attribute. If we expect that the dependent variable depends on certain levels of the attribute in such a way that low levels predict illness, it does not make sense to admit that the level 70 for example predicts illness whereas the level 67 does not predict illness, because the level 67 is also a low level. Consequently, the contraction approach may be useful only if it makes sense to restrict the initial conditions. Another way of contracting the initial conditions would be to retain only the subset of levels $[40,50]$ and to admit that the intermediate subset which provides no prediction is redefined as [51, 87]; this would preserve the intuition that there are low levels of $X$ that predict illness.

Some readers (like one of the anonymous reviewers of the present paper) could ask for the difference between "mere theory disconfirmation" and "the programmatics of fashioning of better theory". Let us suppose that these two cases are out of doubt (if they were, they should not be considered as falsifying cases). Do the two discrepant cases falsify any theory? They falsify the general claim stated in Equation (11). Do we have any theory that implies this general fact? We have only the intuition that low levels should predict illness, if no other conditions that are ignored in the definition of $X$ 's codomain are relevant, and we also admit that other conditions may be relevant, at least at certain levels of $X$. Thus, we should not be surprised by the finding of falsifying cases because we know that the descriptive strategy 
which consists in considering that all relevant initial conditions are taken into account in $X$ 's codomain is naive. Moreover, test scores are not undisputable descriptive values: In a nutshell, the test scores are the names of equivalence classes of multivariate descriptions (usually, the responses to the test items), and the ordering that is imposed on these classes via their numeration (scoring) is a disputable convention, a fact that is not new (Johnson, 1935, 1943) but remains usually ignored (Michell, 1990, 1997, 2000). We cannot discuss the theoretical and methodological consequences of such criticism in this paper (but we do it elsewhere). Thus, if we maintain the intuition that, ceteris paribus, low levels on $X$ should imply illness, and because the ceteris paribus condition is not controlled, contraction may be useful for prediction based both on evidence and intuition if we assume that within the contracted range, which is $[40,50]$, some 'factors' that are relevant out of this range can be neglected within this range. Of course, such reasoning is perfectly inductive (ad hoc) and thus requires further testing. If further tests were to corroborate the contracted statement, there is no need to propose potential relevant factors within this range and then for raising the issue of their empirical description; if these tests falsify it, contraction failed and the issue consists of discovering the relevant factors, which yields expansion. ${ }^{4}$

\subsection{Expansion}

From the point of view of the known initial conditions, any observation unit $\omega$ is described in $M(X)$. For the sake of simplicity, we have chosen our working example because $X$ offers a univariate description. To illustrate the logic of expansion, we will consider only additional descriptions (potential relevant factors) that were ignored in $X$ 's codomain definition, but are nevertheless available in the data (viz., gender, age, and educational level). Thus, considering additional descriptive factors (or dimensions according to Krause, 2010) in the initial conditions yields to considering a multivariate independent variable, that is, a vector of descriptive functions $X_{1} X_{2} \ldots X_{m}$, in which case its codomain is the $m$-ary Cartesian product $M\left(X_{1}\right) \times M\left(X_{2}\right) \times \cdots \times M\left(X_{m}\right)$, where $m$ is the number of factors that serve to describe the observation units' initial conditions.

The idea of expansion as opposed to contraction consists of discovering an independent variable, to be denoted by $X^{\prime \prime}$, that is useful to specify the

\footnotetext{
${ }^{4} \mathrm{An}$ other consequence is that the theoretical and yet descriptive grounds of the test scores could be seriously questioned, but we ignore this way here.
} 
counter-examples with respect to the initial conditions. The dimensionality of the codomain $M\left(X^{\prime \prime}\right)$ is unknown and ranges in $[1,2, \ldots, m-1]$. Let us supposes that $X^{\prime \prime}$ does exists and let $\alpha^{\prime \prime}$ be the subset that contains the images of the counter-examples in $M\left(X^{\prime \prime}\right)$. The updated initial conditions are

$$
\alpha^{\prime}=\alpha \cup\left(M\left(X^{\prime \prime}\right) \backslash \alpha^{\prime \prime}\right),
$$

and the independent variable becomes $X X^{\prime \prime}$. Repairing $\left(\Omega_{n}, \alpha, \beta\right)$ yields $\left(\Omega_{n}, \alpha^{\prime}, \beta\right)$.

Coming back to the example, the two counter-examples share specifically the conjonctive property of being female, more than 83 years old, and not having studied at the secondary educational level. The word "specifically" is crucial here because it is necessary that the remaining observation units do not possess this property. Let $\alpha^{\prime \prime}$ denote this descriptive state- $X^{\prime \prime}$ is a 3 -variate function based on gender, age, and educational level. Thus, the pseudo-general fact (11) may be repaired as the following general fact:

$$
\forall \omega \in \Omega_{154}, \quad\left(X(\omega) \in[40,71] \& X^{\prime \prime}(\omega) \notin \alpha^{\prime \prime}\right) \Rightarrow Y(\omega)=i,
$$

The preceding statement highlights the fact that expansion consists in saving a general statement by isolating its falsifying cases, which consists of adding negative requirements (e.g., not being female and more than 83 years old and having a 'low' educational level) to the initial conditions (e.g., having a low test score or, in other terms, the test score is in [40, 71]). Like contraction, expansion is an inductive solution to the falsifying cases. Does it help improve theory? Not at all because there is no theory to predict the general statement to be saved. Hence, there is no theory to save or improve at all. In our understanding of what a scientific theory is, it is an abstract restatement of natural or experimental, or empirical laws that articulate these laws with parsimony (see Duhem, 1991). Consequently, there is no scientific theory in a field of research if this field contains no law-just intuitions. This is why the main stake of scientific research in psychology consists in discovering genuine laws, and hence, to discover general facts. This is why we believe that the formalization of a general fact deserves to be known by nomothetic researchers, as it is more difficult to look for a general fact if one ignores its defining property (Vautier, 2011). The ability to describe a data set by a statement of form (2) is a good test for deciding whether the data reveals a general fact. 


\subsection{Known cardinality of a general fact}

The known cardinality of an empirical general fact can be simply measured by the number of its relevant observation units: Each time a new observation unit falling into the class $\alpha$ falls into the class $\beta$ (i.e., a positive observation), or each time a new observation unit falling into the class $\bar{\beta}$ falls into the class $\bar{\alpha}$ (i.e., a negative observation), the known cardinality increments of one unit. It could be useful to introduce the following notation:

$$
(\Omega, \alpha, \beta)_{\left[c_{1}, c_{2}\right]},
$$

where $c_{1}$ and $c_{2}$ denote the number of 'positive' and 'negative' observations, respectively, and $c_{1}+c_{2}$ measures the known cardinality.

A general fact repaired by contraction of $\alpha$ to $\alpha^{\prime}$ inherits the cardinality of the equivalence class induced by $\alpha^{\prime}$ on $\Omega_{n}$. When a general fact has been repaired by expansion, two situations have to be distinguished. First, if it can be verified that the remaining observation units do not possess the isolating properties $\alpha^{\prime \prime}$, the cardinality of the repaired fact is that of its predecessor minus the number of counter-examples. But as the descriptive variable used to isolate the counter-examples may be new, which yields the second situation, the descriptive status of the remaining observation units with respect to $X^{\prime \prime}$ is unknown. Consequently, the researcher has to suppose that the remaining observation units do not possess the isolating property $\alpha^{\prime \prime}$. As this supposition is uncertain, the cardinality of the repaired general fact is reset, hence the triplet $\left(\Omega, \alpha^{\prime}, \beta\right)_{[0,0]}$.

\section{Discussion}

In the neo-Galtonian paradigm, researchers investigate summarized properties of collectives of persons, which describe the average subject, that is, nobody in particular. Consequently, individual observations cannot play the role of falsifying cases in this paradigm; this is the reason why statistical $P_{1}$ models hardly match the notion of an empirical law, but work much as interpretative devices interfacing "hard data" and "soft ideas" via conventional operationalization (Vautier, 2011).

The search for empirical laws requires a descriptive language of form $M(X) \times M(Y)$ and a population $\Omega$, from which observations can be madean observation is a basic statement of form $X Y(\omega)=x_{i} y_{j}$. If there is a law 
in $[\Omega, M(X) \times M(Y)]$, it has the form $(\Omega, \alpha, \beta)$, where

$$
\begin{aligned}
& 1 \leq|\alpha| \leq|M(X)| \\
& 1 \leq|\beta|<|M(Y)| .
\end{aligned}
$$

A law is a special relation that can be characterized by the fact that its graph $G$ is a strict subset of $M(X) \times M(Y):^{5}$

$$
G \subset M(X) \times M(Y)
$$

Let us call $|\beta|-1$ the degrees of freedom $(d f \mathrm{~s})$ of the law $(\Omega, \alpha, \beta)$. By definition, if $d f=0$, the law is totally deterministic; otherwise, it is partially deterministic. As an anonymous reviewer of a previous version of this article suggested, one may be attracted by reducing the $d f_{\mathrm{s}}$ of the law. However, we will argue that this line of 'puzzle-solving' is programmatically premature, as it supposes that a law of positive $d f$ s has already been established. It seems not an exaggeration to state that such laws are rare in psychology. Even if one proposes a law with too many $d f_{\mathrm{s}}(d f>1)$, the first task is to test it. If it is easy to find falsifying observations, there are no $d f$ s to reduce because there is no law. Then, the logic of testing suggests the $P_{2}$-researcher to focus on the search for falsifying cases, or at least for a practically sufficient amount of corroborating evidence if no falsifying cases can be found.

When the starting point of a corroborated law is available, the reducing task to improve the granularity (or precision) of prediction will consist in refining the description of the initial conditions, in order to find "nomothetic moderators'. ${ }^{6}$ However, as long as psychological knowledge is framed in the $P_{1}$-language of radical indeterminacy-there is no restriction on the permissible values of any phenomenon given any initial conditions-, such starting points will be lacking. If the proposed law is not trivial, the law is unlikely, and it is likely that it will be falsified, which entails that the main scientific task will be to repair it rather than to restrict its $d f \mathrm{~s}$.

\footnotetext{
${ }^{5}$ The graph of the relation from $M(X)$ to $M(Y)$ as it is known with respect to the available evidence from the sample $\Omega_{n}$ is defined as follows:

$$
G=\left\{\left(x_{i}, y_{j}\right) \mid \exists \omega_{k} \in \Omega_{n}, X\left(\omega_{k}\right)=x_{i} \& Y\left(\omega_{k}\right)=y_{j}\right\},
$$

where $i$ and $j$ index $X$ and $Y$ 's observed values, respectively.

${ }^{6}$ Krause's (2010) approach to the search for sufficient condition causes rejects the neoGaltonian approach to scientific human psychology, but maintains the search for punctual prediction in $M(Y)$.
} 
In such game where the target is a law, any pre-scientific inspiring source is allowed for descriptive attempts, provided the result is a general fact $(\Omega, \alpha, \beta)_{\left[c_{1}, c_{2}\right]}$ with $c_{1}$ and $c_{2}$ as big as possible. Moreover, until no general fact has been discovered, any substantive theory is, by definition, premature as nomothetic theory. As Krause (2010) has stated,

If there is no SCCs [sufficient condition causes] in the subject matter domain of scientific human psychology, then there is no explanatory science of human psychology, because then there cannot properly be said to be any definable psychological causes of human psychological phenomena but, perhaps, only probabilistic psychological predictors of such phenomena. (p. 59)

There is a philosophical argument for not expecting totally deterministic psychological laws: if one admits that a totally deterministic law can be corroborated when observing psychological phenomena in the $M(X) \times M(Y)$ language, one accepts that there is no need for free will in some specific conditions $(\alpha)$. In other terms, such psychological phenomena obey what Searle (1983) calls physical causality as opposed to intentional causality. The case of partial determinacy leaves room for free will as a defining feature of psychological systems, however they can be described.

Suppose a psychological law has been discovered, such that $\beta$ is a subset of several values. Such law acknowledges the irreducible uncertainty of (human) behavior but bounds it within the fixed limits of $\beta$ given $\alpha$. Hence, such scientific knowledge implies that punctual prediction in $M(Y)$ is not expected given the available scientific knowledge. If applied psychologists are to be serving the social demand by proposing better expectations, they cannot argue that their better expectations are valid when applied to single cases. In the domain of psychological assessment, we contend that the $P_{1}$-paradigm allows researchers interested in satisfying the social demand through psychological measurements (or index-numerology according to Johnson, 1943) to establish that quantitative psychological attributes can be measured because measurement is possible without deterministic laws-even without partially deterministic laws (for a more detailed discussion, see Vautier et al., 2012).

As argued by Atlan (1986), the domination of the natural sciences rests on their technical efficiency. The technical efficiency in the social sciences relates to decision making under uncertainty. It can be expected that a psychological science programmed to demonstrate partial determinism of (human) behavior should provoke fierce resistance in the community of social scientists who 
justify its efforts with respect to social utility. But social utility should not be a sine qua non for advancing psychological research. If the scientific endeavor depends to an excessive extent on the social utility of its applications, it seems that the scientific endeavor has to be of the (neo-)Galtonian sort. We advocate room for disinterested research (see Michell, 1997), which entails a publishing space for $P_{2}$-researchers. We would enjoy being able to claim that psychological laws of form (1), or equivalently, relations with graphs of form (17), have been discovered since the historical beginnings of the scientific endeavor in psychology, and that such claims can be qualified for their intrinsic scientific interest. Currently, to deserve publication, research articles have to be (i) socially interesting or (ii) motivated by rationales formulated in a non-descriptive but substantive language to be perceived as theoretically relevant. Taking into account the epistemological limits of these practices, this seems counter effective. To discover psychological laws (i.e., descriptive statements that are true for any individual person in given conditions) we need to be able to develop a nomothetic research programme, and, for this, public knowledge of the existence of these ideas is of paramount importance.

Atlan, H. (1986). ‘̀ tord et à raison : intercritique de la science et du mythe [Enlightenment to enlightenment: Intercritique of science and myth]. Paris: Éditions du Seuil.

Borsboom, D. (2006). The attack of the psychometricians. Psychometrika, $71,425-440$.

Borsboom, D., Cramer, A., Kievit, R. A., Scholten, Z., \& Franic, S. (2009). The end of construct validity. In R. W. Lissitz (Ed.), The concept of validity: Revisions, new directions, and applications (pp. 135-170). Charlotte, NC: Information Age Publishing.

Cronbach, L. J., \& Meehl, P. H. (1955). Construct validity in psychological tests. Psychological Bulletin, 52, 281-302.

Danziger, K. (1987). Statistical method and the historical development of research practice in american psychology. In L. Krger, G. Gigerenzer, \& M. S. Morgan (Eds.), The probabilistic revolution, Vol. 2: Ideas in the sciences (pp. 35-47). Cambridge: MIT Press. 
Danziger, K. (1990). Constructing the subject: Historical origins of psychological research. New York: Cambridge University Press.

Duhem, P. (1991). The aim and structure of physical theory. Princeton, NJ: Princeton University Press.

Granger, G.-G. (1995). La science et les sciences [Science and sciences]. (2nd ed.). Paris: Presses Universitaires de France.

Harré, R. (2004). Staking our claim for qualitative psychology as science. Qualitative Research in Psychology, 1, 3-14.

Hempel, C. (1966). Philosophy of natural science. Englewood Clifs, NJ: Prentice Hall.

Johnson, H. M. (1935). Some neglected principles in aptitude testing. American Journal of Psychology, 47, 159-165.

Johnson, H. M. (1943). Index-numerology and measures of impairment. American Journal of Psychology, 56, 551-558.

Krause, M. S. (2010). Trying to discover sufficient condition causes. Methodology, 6, 59-70.

Krause, M. S. (2011). Statistical signifiance testing and clinical trials. Psychotherapy, 48, 217-222.

Kuhn, T. S. (1996). The structure of scientific revolutions. (3rd ed.). Chicago: The University of Chicago Press.

Lacot, E., Barbeau, E. J., Thomas-Antrion, C., Basaglia-Pappas, S., Parriente, J., Puel, M., \& Vautier, S. (2011). Le TOP 12 : comment s'en servir pour reprer une pathologie du vieillissement cognitif [The TOP 12: How to use it to identify pathological cognitive aging?]. Revue de Neuropsychologie, Neurosciences Cognitives et Cliniques, 3, 273-283.

Lamiell, J. T. (1998). 'Nomothetic' and 'idiographic': Contrasting Windelband's understanding with contemporary usage. Theory $\&$ Psychology, 8, 23-38.

Lamiell, J. T. (2003). Beyond individual and group differences. Thousand Oaks: Sage. 
Lamiell, J. T. (2013). Statisticism in personality psychologists' use of trait constructs: What is it? How was it contracted? Is there a cure? New Ideas in Psychology, 31, 65-71.

Levi, I. (1980). The enterprise of knowledge: An essay on knowledge, credal probability, and chance. Cambridge, MA: The MIT Press.

Lilienfeld, S. O. (2010). Can psychology become a science? Personality and Individual Differences, 49, 281-288.

Maraun, M. D. (1998). Measurement as a normative practice: Implications of Wittgenstein's philosophy for measurement in psychology. Theory $\mathcal{E}$ Psychology, 8, 435-462.

Michell, J. (1990). An introduction to the logic of psychological measurement. Hillsdale, NJ: Lawrence Erlbaum Associates.

Michell, J. (1997). Quantitative science and the definition of measurement in psychology. British Journal of Psychology, 88, 355-383.

Michell, J. (2000). Normal science, pathological science and psychometrics. Theory \& Psychology, 10, 639-667.

Michell, J. (2013). Constructs, inferences, and mental measurement. New Ideas in Psychology, 31, 13-21.

Molenaar, P. C. M. (2004). A manifesto on psychology as idiographic science: Bringing the person back into scientific psychology, this time forever. Measurement: Interdisciplinary Research and Perspectives, 2, 201-218.

Popper, K. R. (1959). The logic of scientific discovery. Oxford England: Basic Books.

Rasch, G. (1960). Probabilistic models for some intelligence tests and attainment tests. Copenhagen: Denmarks Paedagogiske Institut.

Searle, J. R. (1983). Intentionality: An essay in the philosophy of mind. Cambridge, England: Cambridge Univerity Press.

Valéry, P. (1973). Monsieur Teste. Princeton, NJ: Princeton University Press. 
Vautier, S. (2011). The operationalization of general hypotheses versus the discovery of empirical laws in psychology. Philosophia Scientiae, 15, 105122.

Vautier, S. (2013). How to state general qualitative facts in pychology? Quality \& Quantity, 47, 49-56.

Vautier, S., Gillie, R., \& Veldhuis, M. (2011). About validity of conclusions based on multiple linear regression: A commentary on Kupelian et al. (2010). Preventive Medicine, 52, 465.

Vautier, S., Veldhuis, M., Lacot, E., \& Matton, N. (2012). The ambiguous utility of psychometrics for the interpretative foundation of socially relevant avatars. Theory \& Psychology, 12, 810-822. 